\title{
Type Of Drug Interaction Consequence
}

National Cancer Institute

\section{Source}

National Cancer Institute. Type Of Drug Interaction Consequence. NCI Thesaurus. Code C54383.

A category of results of drug adverse reaction or drug interaction. 